\title{
RSD1 Is Essential for Stomatal Patterning and Files in Rice
}

\section{Qi Yu, Liang Chen, Wenqi Zhou, Yanhuang An, Tengxiao Luo, Zhongliang Wu, Yuqi Wang, Yunfeng Xi, Longfeng Yan and Suiwen Hou*}

Key Laboratory of Cell Activities and Stress Adaptations, Ministry of Education, School of Life Sciences, Lanzhou University, Lanzhou, China

Stomatal density is an important factor that determines the efficiency of plant gas exchange and water transpiration. Through forward genetics, we screened a mutant rice stomata developmental defect 1 (rsd1-1) with decreased stomatal density and clustered stomata in rice (Oryza sativa). After the first asymmetric division, some of the larger sister cells undergo an extra asymmetric division to produce a small cell neighboring guard mother cell. Some of these small cells develop into stomata, which leads to stomatal clustering, and the rest arrested or developed into pavement cell. After map-based cloning, we found the protein encoded by this gene containing DUF630 and DUF632 domains. Evolutionary analysis showed that the DUF630/632 gene family differentiated earlier in land plants. It was found that the deletion of RSD1 would lead to the disorder of gene expression regarding stomatal development, especially the expression of stomatal density and distribution 1 (OsSDD1). Through the construction of OsSDD1 deletion mutants by CRISPR-Cas9, we found that, similar to rsd 1 mutants, the ossdd1 mutants have clustered stomata and extra small cells adjacent to the stomata. OsSDD1 and RSD1 are both required for inhibiting ectopic asymmetric cell divisions (ACDs) and clustered stomata. By dehydration stress assay, the decreased stomatal density of $r s d 1$ mutants enhanced their dehydration avoidance. This study characterized the functions of RSD1 and OSSDD1 in rice stomatal development. Our findings will be helpful in developing drought-resistant crops through controlling the stomatal density.

Keywords: stomatal development, stomatal density, RSD1, OSSDD1, dehydration avoidance, rice

\section{INTRODUCTION}

Stomata are small valves in the epidermis of plants for gas exchange between plants and the environment and play essential roles in regulating photosynthesis and water use efficiency (Hetherington and Woodward, 2003). Proper stomatal density and patterning are very important for the growth of plants. There are great differences in stomatal patterning and development processes between monocotyledons and dicotyledons. In Arabidopsis, stomata are constantly generated in different positions of the epidermis during leaf development. The asymmetric entry division of some protodermal cells named meristemoid mother cell (MMC) initiates the stomatal lineage and produces a larger daughter cell called stomatal lineage ground cell (SLGC) and a smaller meristemoid (M). The M can undergo asymmetrically amplifying divisions to renew itself and generate more SLGCs. Then, the 
M converts into the guard mother cell (GMC). The GMC divides equally to form a pair of guard cells (GCs). The SLGCs can differentiate into pavement cells or divide asymmetrically to produce a new $\mathrm{M}$ oriented away from preexisting stomata or stomatal precursors (Bergmann and Sack, 2007).

Most of crops belong to grasses, and they have a great impact on food security (Godfray et al., 2010; Elert, 2014). In contrast to the scattered pattern of Arabidopsis leaves, stomata in graminoid grasses (monocots) are distributed in files. Stomatal development in rice consists of six stages. Epidermal cells that acquired lineage fate undergo an asymmetric entry division to produce two daughter cells, a small cell, and a large sister cell (Stages I and II) (Stebbins and Shah, 1960; Luo et al., 2012; Raissig et al., 2016; Wu et al., 2019; McKown and Bergmann, 2020). Since the absence of a stem-cell-like meristemoid stage in the rice stomatal development, the small cell is named GMC (Stebbins and Shah, 1960; Nunes et al., 2020). The GMC induces the polarization of the subsidiary mother cell (SMC), which then divides asymmetrically to produce a subsidiary cell (SC) and a pavement cell (Stage III and Stage IV) (Cartwright et al., 2009; Facette et al., 2015). After that, GMCs divide symmetrically to produce a pair of GCs (Stage V). Finally, the four-cell stomatal complex is formed (Stage VI) (Stebbins and Shah, 1960).

The stomatal lineage cell fate transformation mechanism has been well studied in Arabidopsis. Three basic helix-loophelix (bHLH) family transcription factors SPEECHLESS (SPCH), MUTE, and FAMA control the consecutive MMC-M-GMC-GC cell fate transitions (Ohashi-Ito and Bergmann, 2006; MacAlister et al., 2007; Pillitteri et al., 2007; Lampard et al., 2008; Chen et al., 2020). These specified cell state transitions require another two paralogous bHLH transcription factors, INDUCER OF CBF EXPRESSION1 (ICE1) and SCREAM2 (SCRM2), to form heterodimers with SPCH, MUTE, and FAMA (Kanaoka et al., 2008). In addition, the cell fate transition from GMC to GC is regulated by FOUR LIPS (FLP) and MYB88, two partially redundant R2R3 MYB transcription factors (Lai et al., 2005; Lee et al., 2014).

Recently, the molecular mechanisms that promote stomatal development in grasses are gradually elucidated. In grasses, the new factors OsSCRs/OsSHRs control the initiation of stomatal lineage cells, and the formation of SCs has been reported recently (Schuler et al., 2018; Wu et al., 2019). The core factors regulating stomatal fate transformation have similar but different functions. OsSPCH1/2 control formation of stomatal files (Raissig et al., 2016; Wu et al., 2019). OsMUTE is expressed in early stage of GMCs and moves to SMC to regulate SC formation. In addition, OsMUTE is involved in the direction of GMC division (Raissig et al., 2017; Wang et al., 2019; Wu et al., 2019). OsFAMA influences SMC division and differentiation of mature stomata (Liu et al., 2009; Wu et al., 2019). OsICE1 and OsICE2 influence the initiation of stomatal lineage, GMC transition, SMC division, and the differentiation of mature stomata (Raissig et al., 2016; $\mathrm{Wu}$ et al., 2019). The OsFLP regulates the direction of GMC division ( $\mathrm{Wu}$ et al., 2019). In addition, an A2-type cyclin; OsCYCA2;1 positive regulates entry division in stomatal file (Qu et al., 2018).
The stomatal patterning in Arabidopsis follows the one-cellspacing rule; that is, two stomatal complexes are separated by at least one non-stomatal cell to ensure a reasonable stomatal density and a proper stomatal patterning in different environmental conditions (Lau and Bergmann, 2012; Dow et al., 2014; Qi and Torii, 2018). Epidermal patterning factors (EPFs) include negative regulators EPF1/2 and EPFL4-6, and a positive regulator EPFL9/STOMAGEN regulates stomatal density (Hara et al., 2007, 2009; Hunt and Gray, 2009; Abrash and Bergmann, 2010; Sugano et al., 2010; Niwa et al., 2013). These ligands bind to the receptor complex consisting of ERECTA family receptor kinase [RLK; ER, ERECTA-LIKE1 (ERL1), and ERL2] and TOO MANY MOUTHS (TMM) (Shpak et al., 2005; Lee et al., 2012, 2015). Downstream of the receptors is a mitogen-activated protein kinase (MAPK) cascade, which is composed of YODA and MKK4/5/7/9 and MPK3/6 to inhibit SPCH activity (Bergmann et al., 2004; Wang et al., 2007; Lampard et al., 2008). The predicted serine protease STOMATAL DENSITY AND DISTRIBUTION1 (SDD1) also negatively regulate stomatal patterning and density by genetically acting upstream of TMM (Berger and Altmann, 2000; Von Groll et al., 2002). The function of SDD1 in dicotyledonous plants is conserved. Overexpression of tomato SchSDD1-like in cultivated tomato plants decreased the stomatal index and density (Morales-Navarro et al., 2018).

The grass stomatal patterning stands in line and also abides by the one-cell-spacing rule that two stomatal complexes are separated by at least one pavement cell. The role of EPFs is conserved in stomatal development. In rice and wheat, the overexpression of OsEPF1/2 and TaEPF1/2 has been shown to increase water use efficiency by reducing stomatal density (Hughes et al., 2017; Caine et al., 2019; Dunn et al., 2019). OsEPFL9 can promote stomatal development, and knocking down OsEPFL9 reduces stomata density in rice (Lu et al., 2019). In addition, BdYODA1 in Brachypodium distachyon involved in maintaining stomatal lineage fate asymmetry and loss of $B d Y O D A 1$ results in large sister cells obtain stomatal fate (Abrash et al., 2018). In maize, the overexpression of ZmSDD1 results in stomatal density decrease of $30 \%$ and enhances the drought resistance (Liu et al., 2015).

In this study, we identified a novel stomatal mutant from EMS mutants' library. This mutant exhibits clustered stomata and reduced stomatal file density and was named as rice stomata developmental defect 1 ( $r s d 1)$. Detailed analysis of stomatal development process indicated that larger sister cell of entry division underwent excessive asymmetric division in rsd1-1. Map-based cloning showed that RSD1 encoded a protein also named REL2, which functions in controlling leaf rolling. The quantitative reverse transcription-quantitative polymerase chain reaction (RT-qPCR) result indicated that the expression of OsSDD1 was significantly down-regulated in $r s d 1$ mutants. Knockout of OsSDD1 produced similar stomatal phenotype with rsd1 mutants, clustered stomata, and extra small cells adjacent to the stomata. OsSDD1 and RSD1 are both required for inhibiting ectopic ACDs and clustered stomata. More importantly, the looss of RSD1 decreased stomatal density and resulted in higher dehydration avoidance. 


\section{MATERIALS AND METHODS}

\section{Plant Materials and Growth Conditions}

Rice (Oryza sativa L. japonica cv. Zhonghua 11, ZH11) was used as the wild type in this study. The $r s d 1-1$ mutant with clustered stomata was screened from the M2 generation of EMS mutant library and then back-crossed into $\mathrm{ZH} 11$ three times prior to use. The rel2 mutant was acquired from Kunming Chen's lab (Supplementary Table 1). The seedlings were grown initially on Murashige and Skoog (MS) medium under 16:8 h, light-dark cycles for 5-7 days and then cultivated in the glasshouse at Lanzhou University (Gansu, China), with a $12 \mathrm{~h}$ photoperiod, $60-80 \%$ relative humidity, and a day/night temperature of $32^{\circ} \mathrm{C} / 22^{\circ} \mathrm{C}$.

\section{Dental Resin Impression and Stomatal Density}

The dental resin impression method was used to screen mutants rsd1-1, rsd1-2, rel2, sdd1-1, and $s d d 1-2$ with clustered stomata. Fully expanded fourth and fifth rice leaves were used to impress the abaxial side, and the detailed impression procedures and stomatal density statistics were performed through methods in our previous report (Luo et al., 2012).

\section{Imaging and Microscopy Analysis}

For confocal imaging, the FM4-64 was captured using a Nikon (A1R+Ti2-E) confocal microscope. The base of the fifth leaf was cut into $0.5 \mathrm{~cm}$ pieces stained in FM4-64 solution. The strain method was performed according to our previous report ( $\mathrm{Wu}$ et al., 2019). Images of the leaf were used for statistical analyses.

\section{Map-Based Cloning of RSD1}

Plants with the clustered stomata were isolated as recombinants from F2 plants of a cross between the rsd1-1 (O. sativa L. japonica cv. Zhonghua 11, ZH11) and 9,311 (O. sativa L. indica) hybrids were selected using dental resin impressions for mapping. The published RM-series rice simple sequence repeat markers ${ }^{1}$ were used to map the mutant gene. The locus was roughly mapped between RM228 and RM590 on the short arm of chromosome 10 by the primary location. Subsequently, the locus was fine mapped onto the $\sim 440 \mathrm{~kb}$ region between two new development markers $\mathrm{X}-02$ and X-08 using 76 homozygote mutants (Supplementary Table 1). The markers were designed by Primer Premier 5.0 and the genomic sequence acquired from the Gramene. The candidate gene RSD1 was identified by sequence analysis of all genes on the region.

\section{Phylogenetic Tree Construction}

The genes containing DUF630 and DUF632 domains were identified from the databases JGI, Marchantia polymorpha (Bowman et al., 2017), Physcomitrella patens (Lang et al., 2018), Arabidopsis thaliana (Lamesch et al., 2012), Medicago truncatula (Young et al., 2011), Solanum lycopersicum (Tomato Genome, 2012), O. sativa (Ouyang et al., 2007), Zea mays

${ }^{1}$ http://www.gramene.org/
(Schnable et al., 2009), B. distachyon (Vogel et al., 2010), Zostera marina (Olsen et al., 2016), and Brachypodium stacei (Gordon et al., 2020) by using the reciprocal BLAST technique with RSD1 protein sequence. The program BLASTP had an $e$-value cutoff of 1-E30. These sequences were further verified using Simple Modular Architecture Research Tool (SMART) protein analyzing software (Letunic and Bork, 2017). Sequences that were confirmed by both methods were used for further analyses. Eventually, the genes from this species were used for phylogenetic analyses in this study. Full-length amino acid sequences were aligned using CLUSTALW2 (Larkin et al., 2007). The neighborjoining (NJ) (Saitou and Nei, 1987) tree was constructed by using the Molecular Evolutionary Genetics Analysis version 5.0 (MEGA 5). The tree nodes were evaluated by bootstrap analysis with 1,000 replicates. Branches with bootstrap values less than $50 \%$ were collapsed. The evolutionary tree is displayed by Interactive Tree of Life ${ }^{2}$ (Letunic and Bork, 2019).

\section{Generation of Mutant Plants by CRISPR/Cas9}

The Vector pBGK032 to construct CRISPR/Cas9 line was performed from our previous report (Wu et al., 2019). The targeting sequences of RSD1 and OsSDD1 were selected (Supplementary Table 2). The designed targeting sequences were inserted into pBGK032 vector to produce CRISPR/Cas9 plasmids as described previously (Wu et al., 2019). The vectors were transformed into rice cultivars $\mathrm{ZH} 11$ as described previously (Nishimura et al., 2006). The transgene lines were extracted genomic DNA and PCR amplification acquired target sequences (Supplementary Table 1). The PCR products were sequenced and analyzed by CRISPR-GE ${ }^{3}$ (Xie et al., 2017). The mutant lines used in our experiments were predicted resulting truncated protein.

\section{Real-Time PCR Analysis}

The method used for extracting the total RNA and RT has been described previously. For quantitative real-time PCR, we used a TB Green Premix Ex Taq (Takara Bio, Inc.) and a StepOnePlus Real-Time PCR System (Applied Biosystems) running a standard programme (Supplementary Table 1). For each real-time PCR experiment, individual samples had three biological replicates per experiment, and all experiments were repeated at least three times.

\section{Dehydration Response Analysis: Water Deficit Shock Treatment}

For the measurement of water loss from leaves, 8 weeks old fully expanded leaves of rice wild-type plants $\mathrm{ZH} 11$ and $r s d 1$ mutants ( $r s d 1-1$ and $r s d 1-2)$ were excised and placed on weighing paper with three replicates. All samples were dried slowly under $22^{\circ} \mathrm{C}$ and $50 \%$ relative humidity. The weight was measured every half hour. The percentage of the sample weight at each time point relative to the initial weight was the water loss weight. Three independent experiments were performed.

\footnotetext{
${ }^{2}$ https://itol.embl.de/

${ }^{3} \mathrm{http}: / /$ skl.scau.edu.cn/
} 


\section{RESULTS}

\section{rsd1-1 Exhibits Clustered Stomata and Reduced Stomatal Files}

By screening EMS mutants' library generated from $O$. sativa japonica cultivar ZH11, we identified a stomatal mutant with clustered stomata, decreased stomatal density, and files (Figures $\mathbf{1 A}, \mathbf{B}, \mathbf{K}-\mathbf{N}$ ). We named the mutant as rice stomata developmental defect 1 ( $r s d 1)$. In wild type, stomata strictly comply with the principle of "one-cell-spacing rule," that is, two stomatal complexes had to be separated by at least one pavement cell (Figures 1A,C). In $r s d 1$-1, clustered stomata were observed, and some of them co-use a SC (Figures 1B,D). In addition, we observed small cells neighboring some stomata in stomatal file (Figures 1B,E-G). Some of the small cells were able to induce an extra SC, but they would not develop into mature stomata (Figure 1E). Some of small cells seemed to obtain the fate of pavement cell with lobes (Figures 1F, G). Occasionally, we observed that some GMCs exited stomatal lineage before or after inducing unilateral or bilateral SCs (Figures $\mathbf{1 H}-\mathbf{J}$ ), suggesting that $R S D 1$ is required for promoting GMC to differentiate into mature stomata. Statistical analysis revealed that the percentage of clustered stomata or extra small cell neighboring stomata significantly increased (Figure 10). We observed that the stomatal density and stomatal file density of rsd1-1 decreased in the same position and phyllotaxis of leaf blade (Figures $1 \mathbf{K}-\mathbf{N})$. Together, these results indicated that RSD1 regulates stomatal distribution pattern and density.

\section{$R S D 1$ Is Essential for the Differentiation of Large Sister Cells Into Pavement Cell to Establish the Stomatal Patterning}

The stomatal patterning and density of grasses are established in a very small area at the base of the leaf. Stomatal development in grasses consists of six stages (Figures 2A-F) (Luo et al., 2012; Raissig et al., 2016; Wu et al., 2019). At stage II, the stomatal lineage cells initiate entry division to generate a smaller GMC and a larger sister cell that will differentiate into a pavement cell (Stebbins and Shah, 1960; Raissig et al., 2016; Wu et al., 2019). The direct differentiation of large sister cells into pavement cells determines the establishment of stomatal patterning in stomatal files.

The stomatal development stages of $r s d 1-1$ were observed. The stomatal development of $r s d 1$ mutant at stages $\mathrm{I}$ and II were regular (Figures $\mathbf{2 G}, \mathbf{H}, \mathbf{O}, \mathbf{P}$ ). At stage III, some of the larger sister cells underwent an extra asymmetric division to produce an extra small cell neighboring GMC (Figures 2I,Q). At stage IV, a large number of extra small cells neighboring GMC were produced (Supplementary Figure S1). We observed that some SCs flanked a GMC and its neighboring extra small cell (Figure 2J). Occasionally, we also observed that the GMCs failed to induce SC formation (Figure 2R). At stage $\mathrm{V}$, a few extra small cells neighboring GMC can divide equally to form paired GCs, resulting in clustered stomata (Figure $2 \mathbf{K}$ ), and the rest of the extra small cells neighboring GMC exit stomatal linage (Figure 2M). In addition, the arrested GMCs were occasionally observed (Figure 2S). At stage VI, the abnormal stomata differentiated into mature stomata and form disrupted pattern (Figures 2L,N,T). These observations indicated that RSD1 is required to prevent ACD in large sister cells' reentry stomatal lineage.

\section{Map-Based Cloning of RSD1 Gene}

Our genetic analysis showed that the clustered stomata of $r s d 1$ 1 were caused by a single recessive mutation. To identify the mutated gene, the map-based cloning strategy was used, and the candidate gene locus was mapped in a $440 \mathrm{~kb}$ region between two newly developed molecular markers (X-02 and $\mathrm{X}-08$ ) in chromosome 10 (Figure 3A). Using whole-genome sequencing, we found a $1 \mathrm{bp}$ deletion at the fourth exon of LOC_Os $10 g 41310$, resulting in a premature transcription termination (PTT) (Figure 3B). The rice genome contains only one copy of the RSD1 gene, which is predicted to encode a protein consisting of 767 amino acid residues. This gene is also named Rolled and Erect Leaf 2 (REL2), which is involved in the control of leaf rolling in rice (Yang et al., 2016).

To confirm whether the stomatal phenotypes of rsd1-1 were caused by the mutation of LOC_Os10g41310, we created an additional frameshift mutation that is $1 \mathrm{bp}$ insertion in the first exon, resulting in a PTT by clustered, regularly interspaced short palindromic repeats-associated nuclease 9 (CRISPR/Cas9). We named this mutant rsd1-2 (Supplementary Figure S2). It exhibited similar stomatal phenotypes with $r s d 1-1$ (Figures 3C,D,I). In addition, similar to $r s d 1-1$ and $r s d 1-2$, the allelic mutant rel2 produced clustered stomatal and small cell neighboring stomata (Figures 3E,F,I), and the stomatal density also decreased (Figure 3H). F1 generation plants of a cross between $r s d 1-1$ and $r s d 1-2$ show similar phenotypes with $r s d 1$ mutants (Figure 3G). These results indicated that the mutation of the LOC_Os10g41310 is responsible for stomatal development defects of $r s d 1$ mutants.

\section{Evolutionary Analysis of RSD1 in Plants}

RSD1 encodes a protein containing DUF630 and DUF632 domains (Supplementary Figure S4). The BLASTp analysis indicated that there was no homolog of RSD1 in green alga Coccomyxa subellipsoidea. In liverwort $M$. polymorpha, there was only one copy of RSD1. In moss $P$. patens, there were four putative paralogs. In dicots and monocots, we found lots of homologous genes contained DUF630 and DUF632 domains, such as $M$. polymorpha (1 sequence), $P$. patens (4 sequences), A. thaliana (15 sequences), $M$. truncatula (17 sequences), S. lycopersicum (11 sequences), O. sativa (20 sequences), Z. mays (21 sequences), B. distachyon (17 sequences), and B. stacei (18 sequences). In order to determine the evolutionary relationship between the RSD1 proteins with different species, we performed multiple sequence alignment and generated an NJ phylogenetic tree for RSD1 proteins from nine land plants (Figure 4). These genes were divided into clade A containing 45 proteins and clade B containing 74 proteins. Phylogenetic analysis indicated that the homologous genes diverged early in the evolution of land plants (Figure 4). Pairwise sequence alignment revealed that RSD1 shows $51.1 \%$ amino acid sequence identity with Arabidopsis AtNRG2, which plays a key role in nitrate regulation 

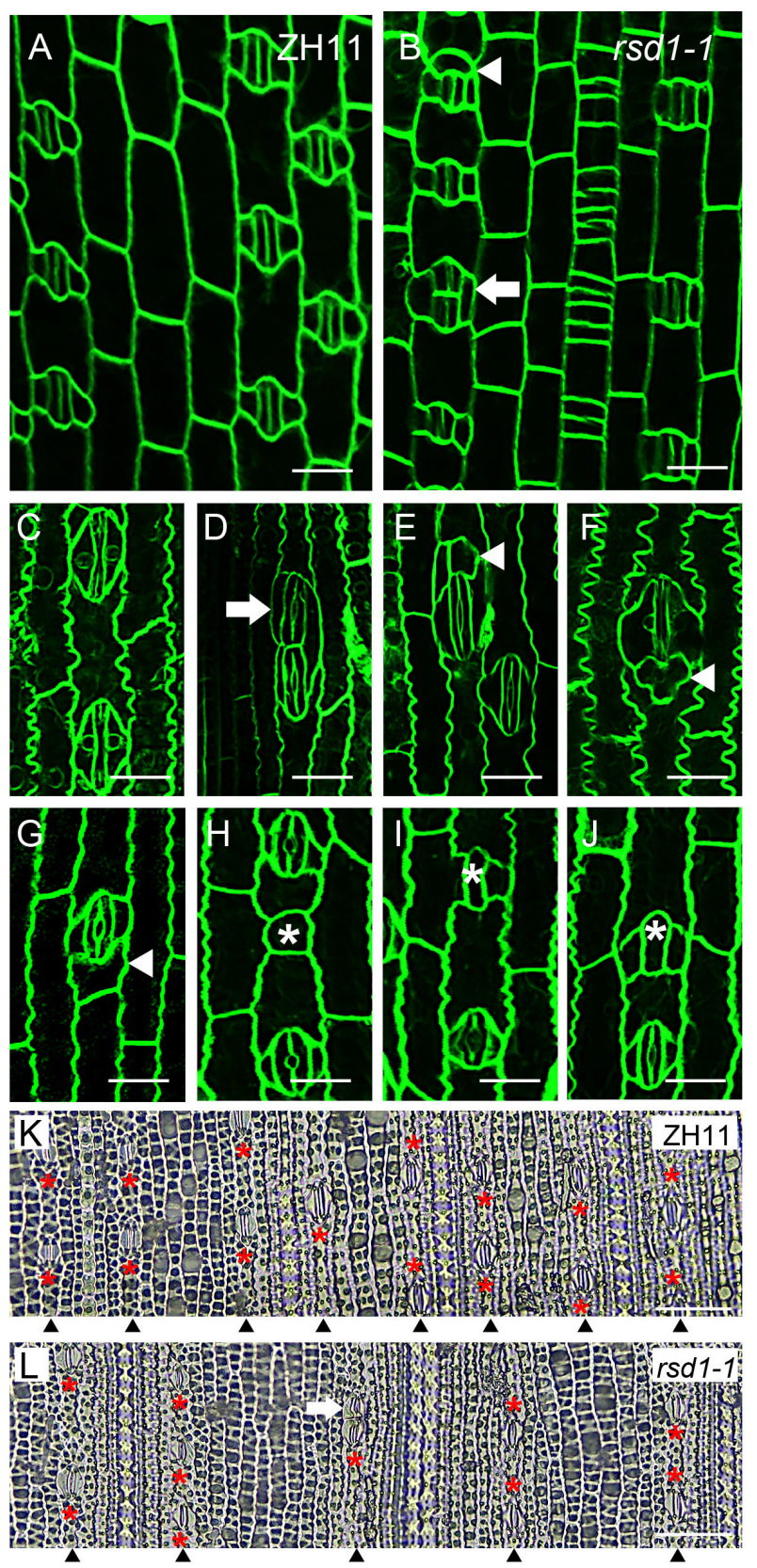

M

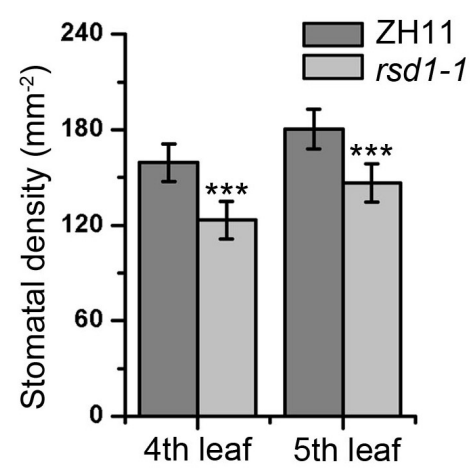

$\mathrm{N}$

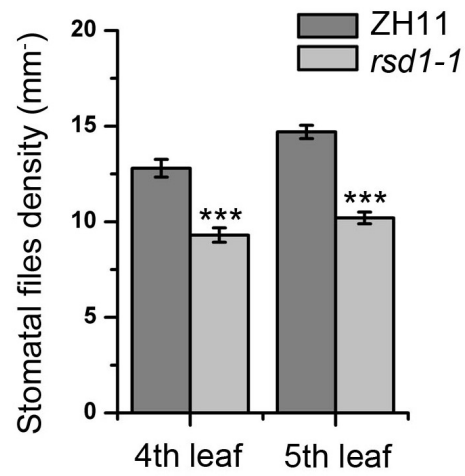

\section{0}

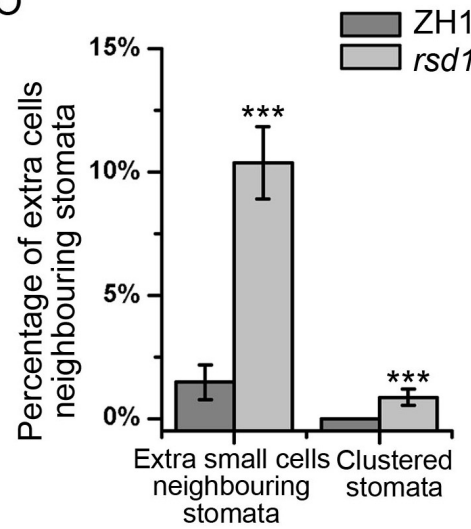

FIGURE 1 | rsd1-1 mutant showed clustered stomata and an extra small cell neighboring stoma. (A) Developing stomata of ZH11. (B) Developing stomata of rsd1-1. (C) Mature stomata of ZH11. (D) Two-cluster stomata sharing one subsidiary cell. (E) A stoma with three subsidiary cells and a small cell. (F) Five-cell stomatal complex. (G) An extra small cell neighboring stoma. (H) A small cell arrested in GMC stage. (I) Arrested GMC with one subsidiary cell. (J) Arrested GMC with both sides of subsidiary cell. Bars, $10 \mu \mathrm{m}$. The white arrowheads indicated ectopic extra small cells neighboring stomata. The white arrows indicated clustered stomata. The white asterisks indicated arrested GMCs. The red asterisks indicated stomata. (K) The stomatal files of ZH11. (L) The stomatal files of rsd1-1. The white arrowheads indicated stomatal files. Bars, $50 \mu \mathrm{m}$. (M) Quantification of the stomatal density at mature stage. (N) Quantification of the stomatal files at mature stage. (O) Quantification of the extra small cells neighboring stomata and clustered stomata at the fifth leave. The error bars indicated the mean \pm SEM, $n=20$; ${ }^{\star \star \star} P<0.001$ by Student's $t$-test.

(Xu et al., 2016). Another gene APSR1 in this family is required for root meristem maintenance (Gonzalez-Mendoza et al., 2013). These limited results indicated that this gene family had important functions in the process of plant growth and development, and the exploration of the gene function of this new gene family would be able to understand the mechanism of plant growth and development.

The number of DUF630/632 family genes in angiosperm is more than liverwort and moss remarkable (Figure 4), indicating that this gene family is growing in richness. The high 

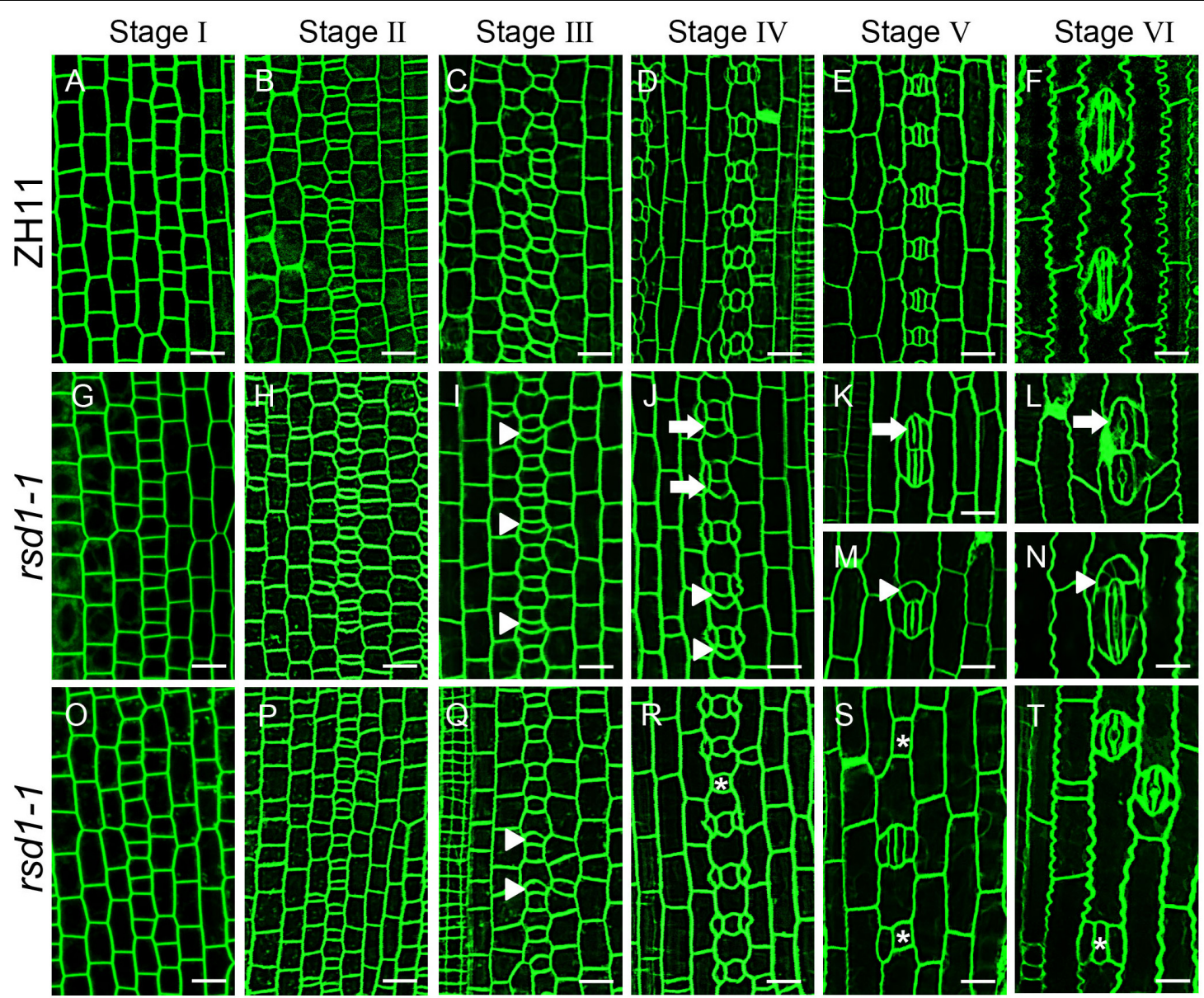

FIGURE 2 | Stomatal developmental process in rsd1-1 mutant. (A-T) Confocal images of six main stomatal developmental stages in rsd1-1 mutant. There was no significant difference between rsd1-1 and ZH11 at stage I and stage II (A,B, G,H,O,P). Extra divisions in large sister cells resulting in extra small cells neighboring GMCs at stage III in rsd1-1 mutant (I,Q). Two-cluster GMCs sharing one subsidiary cell and arrested GMC at stage IV in rsd1-1 mutant (J,R). Clustered stomata, five-cell stomatal complex and arrested GMCs at stage V in rsd1-1 mutant (K,M,S). Morphogenesis and differentiation of clustered stomata, five-cell stomatal complex and arrested GMC at stage VI in rsd1-1 mutant (L,N,T). The white arrowheads indicated ectopic extra small cells neighboring stomata. The white arrows indicated clustered stomata. The asterisks indicated arrested GMCs. Bars, $10 \mu \mathrm{m}$.

number of DUF630/632 family genes in angiosperm indicated that DUF630/632 gene duplication might be important, which was associated with their abundance of function. In marine angiosperm $Z$. marina, the stomatal development genes $(S P C H$, MUTE, FAMA, FLP, TMM, SDD1, EPF1, EPF2, EPFL9) are absent, consisting of its phenotype without stomata (Olsen et al., 2016). However, Z. marina possesses two RSD1 genes (Supplementary Figure S3). These results suggest that RSD1 is not only related to stomatal development, but also is important for plant growth.

\section{RSD1 Is Required for Proper Expression of Stomatal Development-Related Genes}

The RSD1/REL2 is dominantly expressed in the younger leaf blades (Supplementary Figure S5; Yang et al., 2016), suggesting that this gene is involved in stomatal development.

To investigate the relationship between RSD1 and stomatal development, we detected the transcript abundance of 18 important stomatal development genes by RT-qPCR in the base of young leaves in $r s d 1-1$ (Figure 5). The expression of OsMUTE, OsEPF1, and OsSDD1 was down-regulated in $r s d 1$ 1 mutant, whereas the expression of OSICE1 and OsEPFL9 was upregulated (Figure 5). There were no significant changes in other stomatal development genes (Figure 5). The expression of OsMUTE and OsSDD1 was also significantly decreased in $r s d 1-2$ (Supplementary Figure S6). Most small cell neighboring stomata cannot develop into stomata, and some of the GMCs arrested in the mutants. Thus, we suspected that these cells may not express OsMUTE, which may lead to the downregulation of OSMUTE in the $r s d 1$ mutants. These results suggest that $R S D 1$ is required for proper expression of stomatal development-related genes.

\section{OsSDD1 Mutants Exhibit Similar Stomatal Phenotype to rsd1}

The significant downregulation of OSSDD1 in $r s d 1$ mutants led us to investigate the role of $O S S D D 1$ in stomatal development. There 
A

$\mathrm{n}=48$

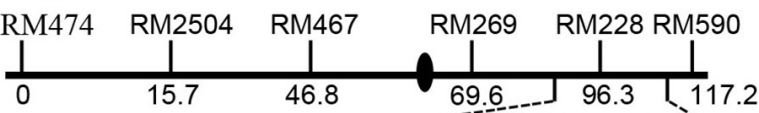

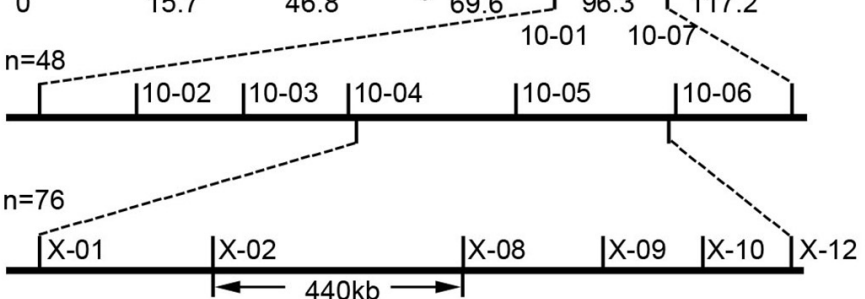

B

LOC_Os10g41310

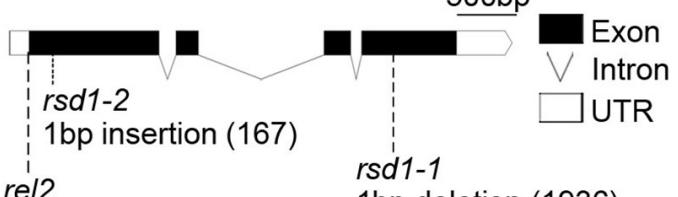

1 bp deletion (1936)

4bp deletion (-92 to -89)

$\mathrm{G}(8) \rightarrow \mathrm{A}$ (Cys $\rightarrow \mathrm{Tyr})$

$\mathrm{G}(40) \rightarrow A(\mathrm{Val} \rightarrow \mathrm{Met})$
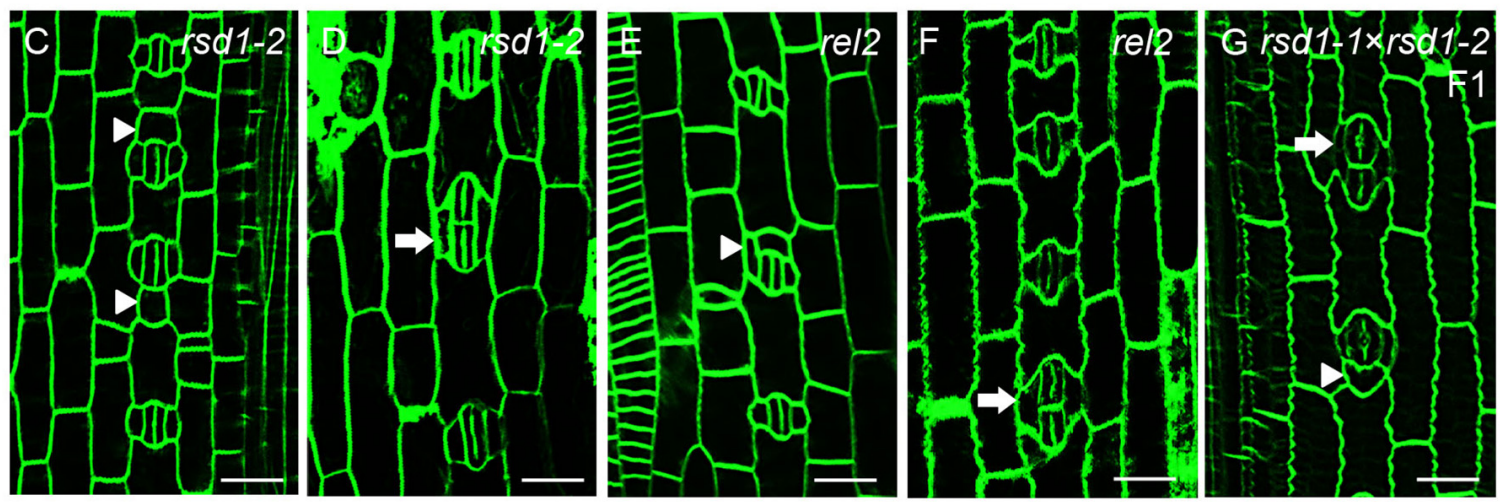

$\mathrm{H}$

\section{I}
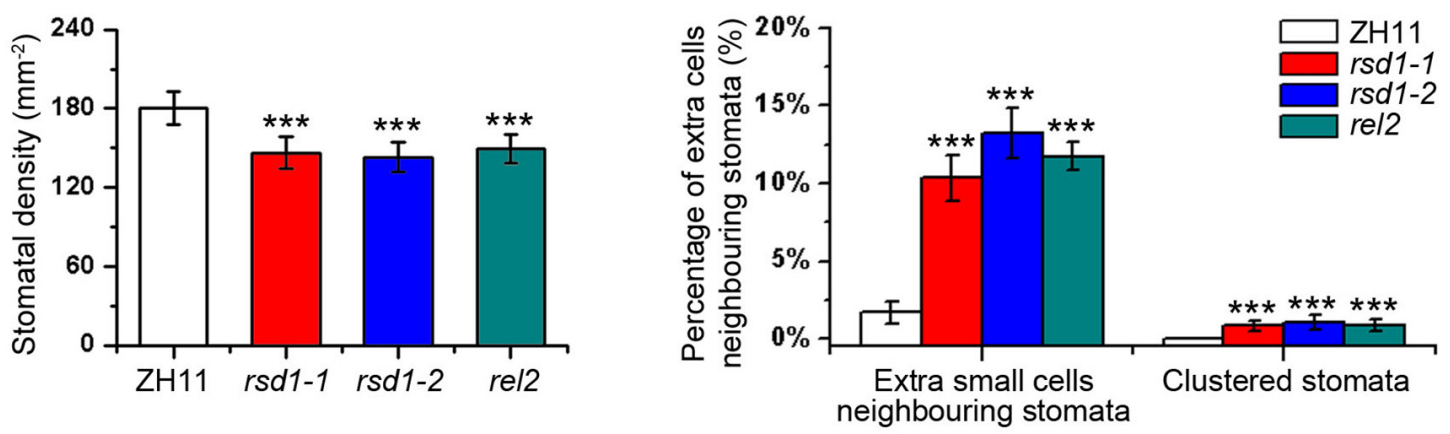

FIGURE 3 | Map-based cloning of rsd1-1 and the phenotype of rsd1-1 allelic mutants rsd1-2 and rel2. (A) RSD1 was localized between X-02 and X-08 on chromosome 10. $n$ represents sample size. (B) Gene structure and mutant sites of candidate gene LOC_Os10g41310 (RSD1). Black boxes indicated the exons, lines indicated the introns, and white boxes represented untranslated region (UTR). The rsd1-1 had a 1 bp deletion in the fourth exon. The rsd1-2 had a 1 bp insertion in the first exon. The rel2 had a 4 bp (GGAG) deletion in 5'-UTR and two mutations (G to A and G to A) in the first exon. (C-G) Confocal images of developing stomata in rsd1-2, rel2, and F1 generation plant of a cross between rsd1-1 and rsd1-2. (C) An extra small cell neighboring stoma in rsd1-2. (D) Two-cluster stomata sharing two subsidiary cells in rsd1-2. (E) An extra small cell neighboring stoma in rel2. (F) Two-cluster stomata sharing two subsidiary cells in rel2. (G) An extra small cell neighboring stoma and clustered stomata in F1 generation plant of a cross between rsd1-1 and rsd1-2. Bars, $10 \mu \mathrm{m}$. The white arrowheads indicated ectopic extra small cells neighboring stomata. The white arrows indicated clustered stomata. (H) Quantification of the stomatal density at mature stage. (I) Quantification of the clustered stomata and extra small cells neighboring stomata at the fifth leaf. The error bars indicated the mean \pm SEM, $n=10$; ${ }^{\star \star \star} P<0.001$ by Student's $t$-test.

was one copy of SDD1 in rice named OSSDD1 (Supplementary Figure S7). To explore the phenotype of OsSDD1, CRISPR/Cas9 genome editing was performed, and we obtained two mutants named ossdd1-1 and ossdd1-2 (Supplementary Figure S8). In ossdd1-1, 1 bp was inserted at nucleotide position between 131 and 132, resulting in a PTT. In ossdd1-2, the 131st nucleotide was deleted, which also led to a PTT (Supplementary Figure S8). We then observed the stomatal phenotype in mature leaves of $Z H 11$, ossdd1-1, and ossdd1-2 (Figures 6A-D). Similar to $r s d 1$ mutants, both ossdd1-1 and ossdd1-2 exhibited clustered stomata and extra small cell neighboring stomata (Figures 6B-D,J). In addition, we observed the stomatal development process in ossdd1-1 (Figures 6E-H). Similar to $r s d 1$, an extra asymmetric division was observed in some larger sister cells of the ossdd 1 mutants at stage III, producing an extra small cell neighboring GMC (Figure 6E). A few of extra small cells neighboring GMC could induce the 


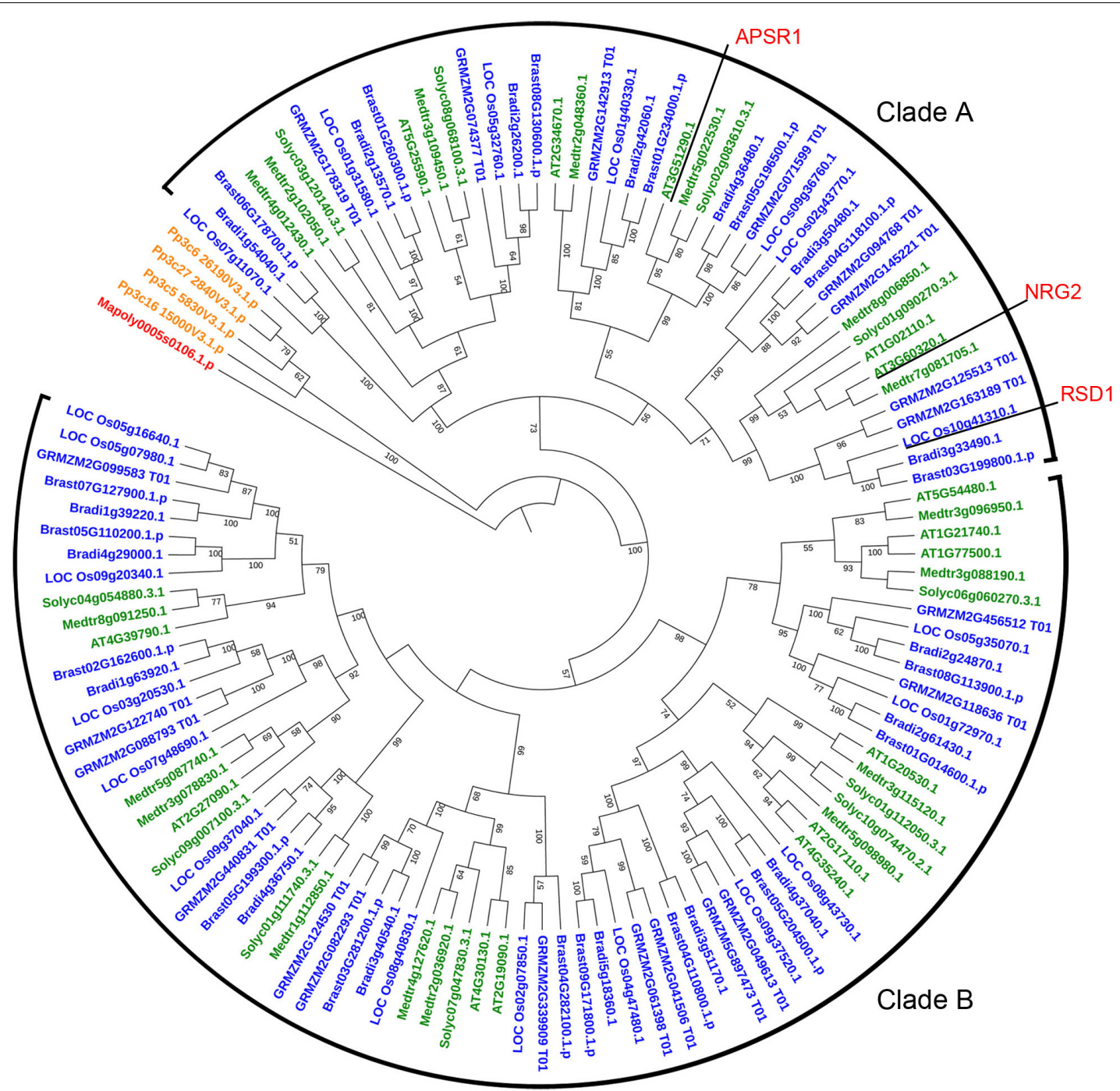

FIGURE 4 | Phylogenetic relationship of proteins containing DUF630 and DUF632 domain of RSD1 in 9 land plants. The protein sequences for constructing phylogenetic tree were identified from the databases Phytozome V12 by using the reciprocal BLAST technique with rice RSD1 protein sequence. The phylogenetic tree was constructed by the NJ method in MEGA 5. Bootstrap values for 1,000 replicates are given in nodes as percentages. Amino acid sequences were used from Marchantia polymorpha (1 sequence), Physcomitrella patens (4 sequences), Arabidopsis thaliana (15 sequences), Medicago truncatula (17 sequences), Solanum Iycopersicum (11 sequences), O. sativa (20 sequences), Zea mays (21 sequences), Brachypodium distachyon (17 sequences), and Brachypodium stacei (18 sequences).

formation of SC at Stage V (Figure 6G) and may finally divide equally to form paired GCs, resulting in clustered stomata at stage VI (Figure 6H). The stomatal density of ossdd1 mutants was slightly increased (Figure 6I), but the stomatal files had nothing different with ZH11 (Supplementary Figure S9). These observations suggested that $O S S D D 1$ is required for restricting ectopic ACDs and clustered stomata but not needed for stomatal file density in rice.

\section{rsd1 Mutants Enhance the Dehydration Avoidance}

As stomatal density was decreased in $r s d 1$ mutants (Figure $\mathbf{1} \mathbf{M}$ ), we performed a dehydration shock stress assay to determine the role of RSD1 in water deficit. In $\mathrm{ZH} 11$, the fresh weight was reduced to $56.62 \%$ after $2 \mathrm{~h}$ of water deficit shock. Compared with $\mathrm{ZH} 11$, the water loss rate of $r s d 1$ mutants was significantly lower. The fresh weight of $r s d 1-1$ and $r s d 1-2$ was reduced to 65.57 and $67.94 \%$, respectively, under the same treatment (Figure 7A). This result suggested that $R S D 1$ is required for regulating water loss by modulating stomatal density.

\section{DISCUSSION}

Stomata are key determinants of the trade-off between photosynthetic carbon fixation and water transpiration (Franks and Farquhar, 1999). Grasses with lower stomatal 


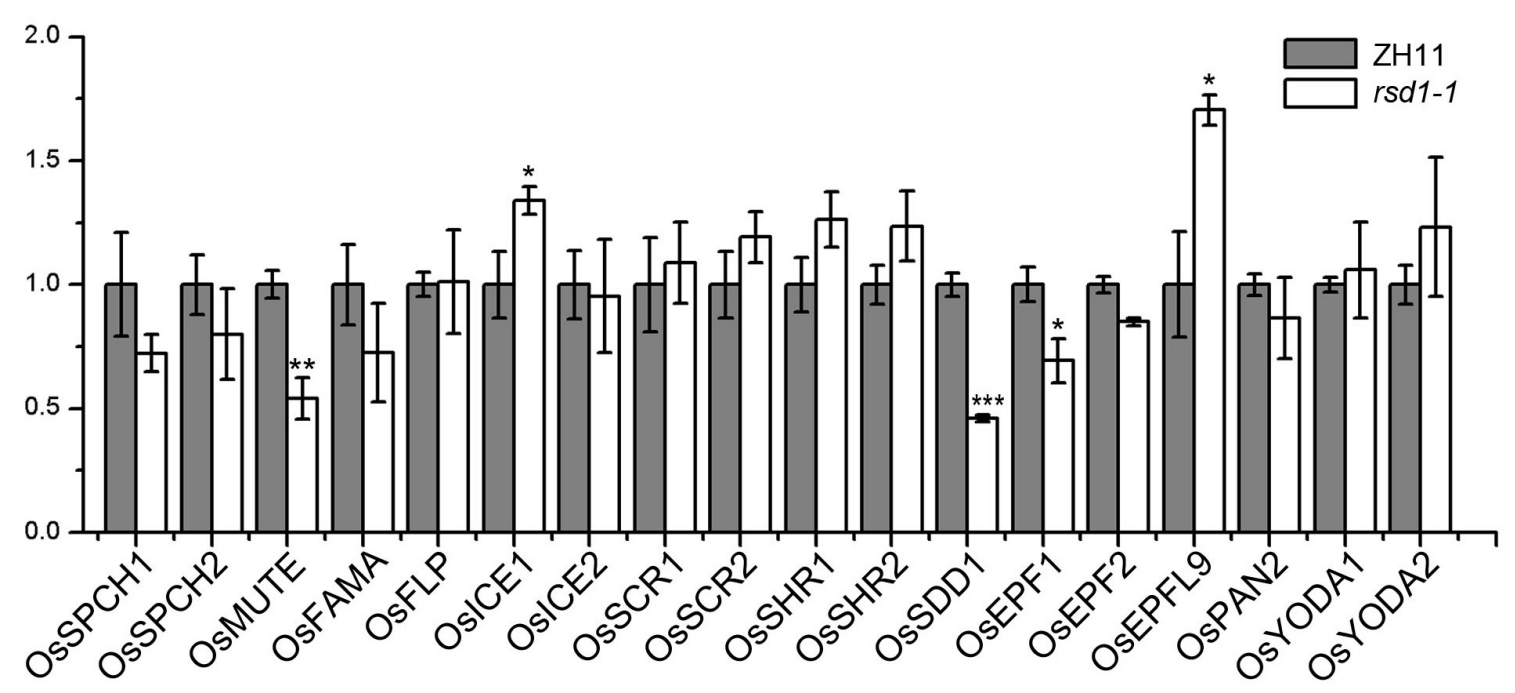

FIGURE 5 | Expression analysis of stomatal development regulation genes in rsd1-1 mutant. Analyzing the expression of stomatal development relative genes in rsd1-1 mutant by RT-qPCR. OsUBQ10 was used as the internal control. The error bars indicated mean $\pm \mathrm{SD}, n=3 ;{ }^{\star \star \star} P<0.001$. ${ }^{\star \star} 0.001<P<0.01$. ${ }^{*} 0.01<P<0.05$ by Student's $t$-test.

density have higher water use efficiency and greater drought tolerance than other species (Hughes et al., 2017). However, the detailed mechanisms of stomatal development in grasses were still very poorly understood. By forward genetic approach, we found $R S D 1$, a new stomatal patterning regulatory gene, was required for inhibiting clustered stomata and promoting stomatal density (Figure 7B). Knockout of OsSDD1 also produced clustered stomata and extra small cells adjacent to the stomata. OsSDD1 and RSD1 are both required for inhibiting ectopic ACDs and clustered stomata (Figure 7C).

In grasses, stomatal patterning is established when each stomatal precursor in a cell file divides once asymmetrically in the same orientation to produce a GMC (Facette and Smith, 2012). Therefore, there may be two ways to produce clustered stomata. One is the disruption of the division direction in neighboring stomatal precursors; another is the reentry of stomatal development in large sister cells to produce a stoma neighboring preexisting stoma. The $r s d 1$ mutants produce clustered stomata. Closer observation of stomatal development stages in the mutants showed that the direction of entry division was not affected. We found that the larger sister cell underwent an asymmetric division neighboring preexisting stoma or GMC, suggesting that the RSD1 is required for inhibiting reentry division in larger sister cells. Similar causes of clustered stomatal were also observed in ossdd1 mutants, suggesting that OsSDD1 is also involved in preventing larger sister cells from dividing asymmetrically and promoting the differentiation of larger sister cells into pavement cells. In addition, some GMCs arrested and failed to differentiate into GC in $r s d 1$ mutants, suggesting a role of $R S D 1$ in promoting GMC to differentiate into mature stomata. These results suggest that $R S D 1$ promoted cell differentiation of both large sister cell and GMC.

The molecular mechanism of clustered stomata in grasses is still unknown. In Arabidopsis, AtSPCH and AtICE1/AtSCRM2 coordinately established stomatal fate (MacAlister et al., 2007; Kanaoka et al., 2008). When they accumulated, additional stomata will be produced, and all epidermal cells will be turned into stomata in extreme conditions (MacAlister et al., 2007; Kanaoka et al., 2008). The upstream MAPK and receptor ligand signals ensure that additional stomata are not produced by limiting the accumulation of fate determination transcription factors (Bergmann et al., 2004; Lampard et al., 2008; Putarjunan et al., 2019). In grass plants, it is found that overexpression of stomatal fate factor $S P C H 1 / 2$ in $B$. distachyon could induce additional cell division in epidermal cells (Raissig et al., 2016; Wu et al., 2019). In POSTECH insertion mutant line of osspch2, the clustered stomata had been observed (Liu et al., 2009). Overexpression of ICE1 and SCRM2 produced only a small amount of extra cell division, whereas Ubipro:BdICE $1^{\text {scrmD }}$ produced clustered stomata (Raissig et al., 2016). In addition, the absence of $B d Y O D A 1$ leads to the disorder of cell fate in stomatal files, which results in clustered stomata (Abrash et al., 2018). In the rsd1-1 mutant, the expression of ICE1 and EPFL9 was slightly up-regulated, which might be one of the reasons of clustered stomata.

AtSDD1 is expressed in pseudo-meristem cells and GMCs, and the stomatal density of the mutant with functional deletion increased by 2-4 times and clustered, and its overexpression inhibited stomatal differentiation and decreased stomatal density (Berger and Altmann, 2000; Von Groll et al., 2002). Its function is to regulate stomatal development in the upstream of TMM but independent of EPFL gene family (Von Groll et al., 2002). Its protein function is conserved to a certain extent and has a significant effect on stomatal density in tomato and maize (Liu et al., 2015; Morales-Navarro et al., 2018). However, the function of OsSDD1 in stomatal development is still unclear in rice. By constructing the knockout lines, we found that the ossdd1 mutants have clustered stomata and extra small cells 

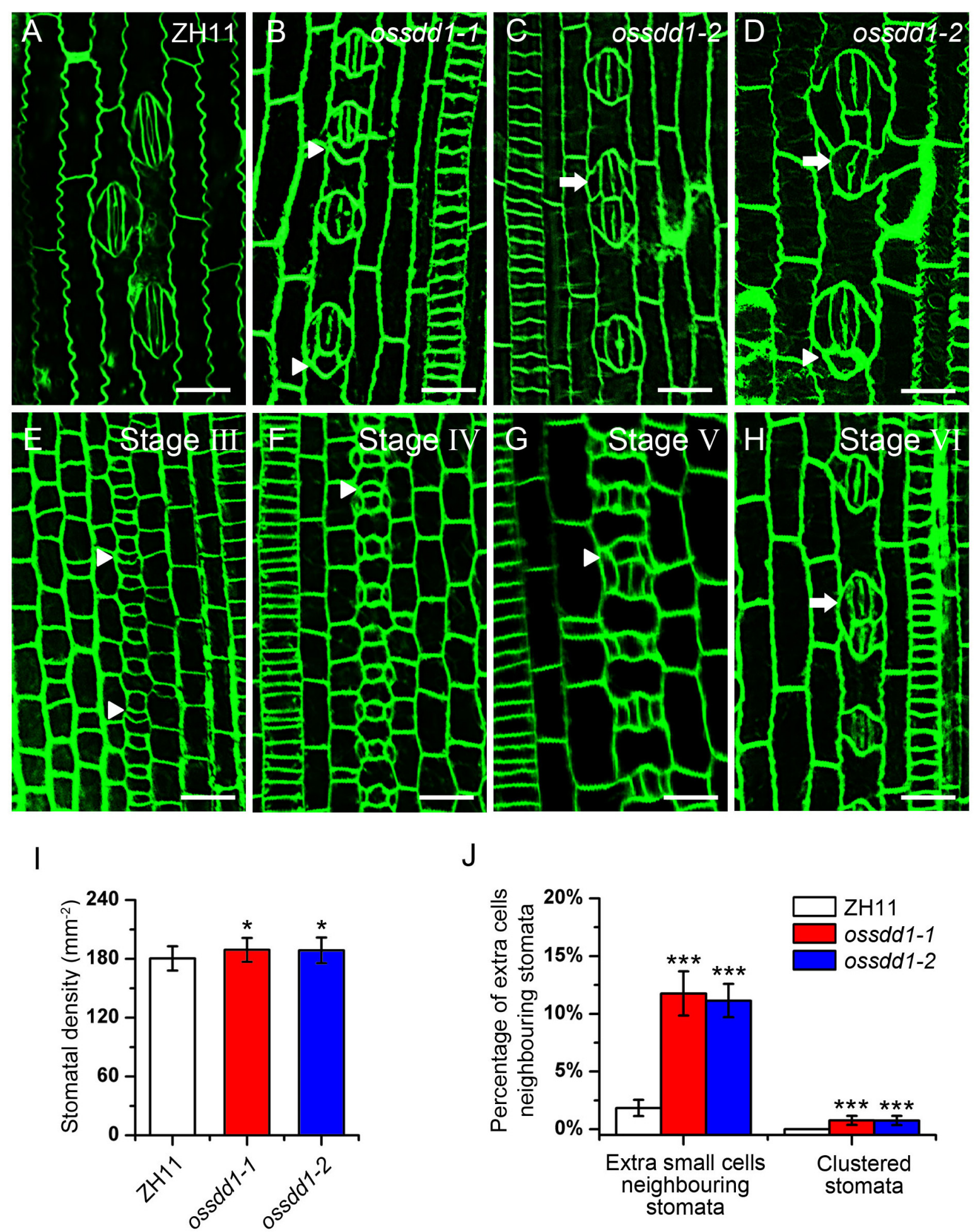

FIGURE 6 | Phenotypic analysis of ossdd1-1 and ossdd1-2 mutants. (A) Mature stomata of ZH11. (B) An extra small cell neighboring stoma in ossdd1-1. (C) Two-cluster stomata with four subsidiary cells in ossdd1-2. (D) Five-cell stomatal complex and ectopic stomatal patterning in ossdd1-2. (E) The extra division of large sister cell resulting in an extra small cell neighboring GMC at stage III in ossdd1-1. (F) GMC with a small cell at stage IV in ossdd1-1. (G) Stomata with a small cell sharing SC at stage V. (H) Morphogenesis and differentiation of clustered stomata at stage VI. Bars, $10 \mu \mathrm{m}$. The white arrowheads indicated extra small cells neighboring stomata. The white arrows indicated clustered stomata. (I) Quantification of the stomatal density at mature stage. (J) Quantification of the clustered stomata and extra small cells neighboring stomata at the fifth leave. The error bars indicated the mean $\pm \mathrm{SEM}, n=10$; ${ }^{\star \star \star} P<0.001 ;{ }^{*} 0.01<P<0.05$ by Student's t-test. 
A

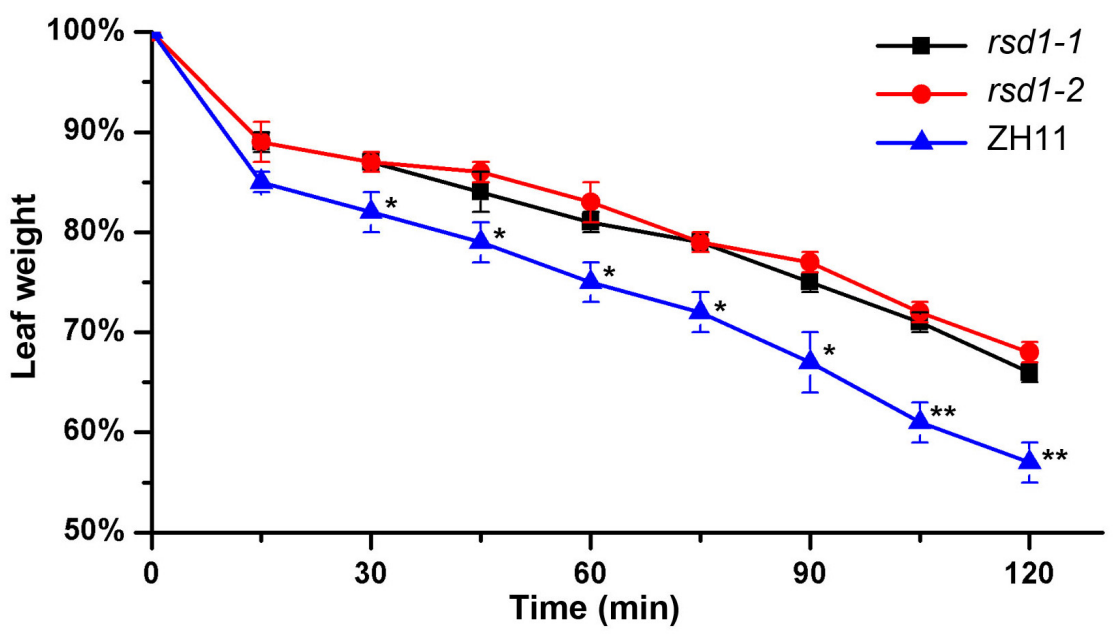

B $\quad \mathrm{ZH} 11$

rsd1
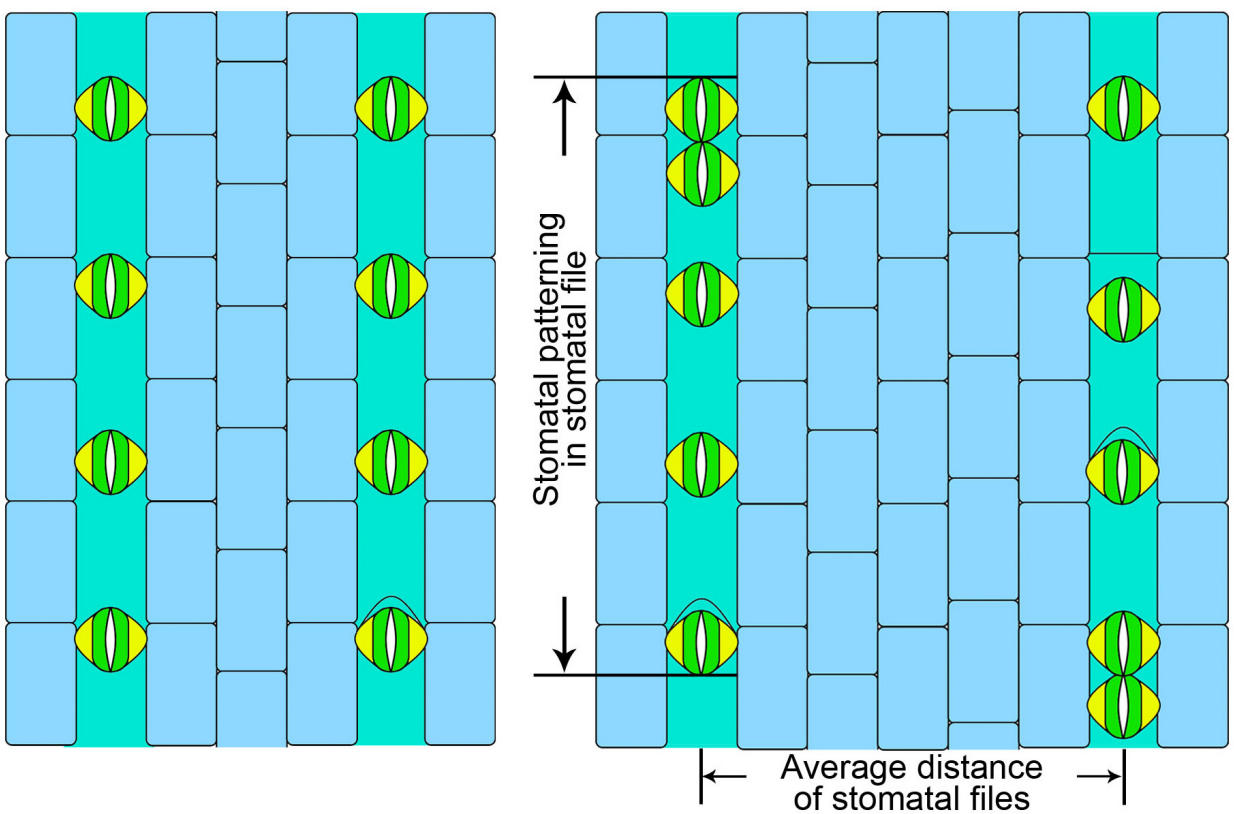

C

Stage I

Stomatal file
specification

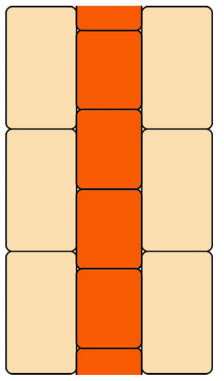

Stage II

RSD1 ?

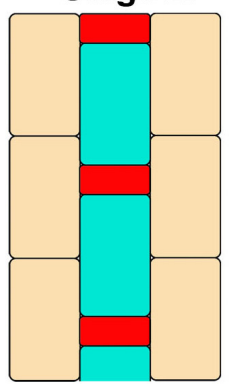

\section{Larger sister cell} formation

RSD1

OsSDD1

FIGURE 7 | The rsd1 mutants increase dehydration avoidance, and the model showed RSD1 and OSSDD1 regulating stomatal development. (A) Water loss in 8 weeks old detached leaves at different time points with three replicates. Three independent experiments were performed with similar results. The error bars indicated the mean \pm SEM, $n=3$; ${ }^{*} 0.001<P<0.01,{ }^{*} 0.01<P<0.05$ by Student's $t$-test. (B) The stomatal patterning in ZH11 and rsd1 mutants. (C) RSD1 and OsSDD1 required at early stage of stomatal development. 
neighboring stomata (Figures 6B-D). The phenotype of ossdd1 mutants is similar to $r s d 1-1$. OsSDD1 and RSD1 are both required for inhibiting ectopic ACDs and clustered stomata.

In grasses, stomata are always arranged parallel and adjacent to leaf veins (Zwieniecki and Boyce, 2014; Nunes et al., 2020). The density of stomatal files was different in different development stages, species, or growth conditions (Stebbins and Shah, 1960). Therefore, the density of stomatal files is the key factor for stomatal density. The grass SHR/SCR is a common module that not only controls vein development and Kranz anatomy in maize (Slewinski et al., 2014; Hughes et al., 2019) but also regulates stomatal development in rice (Kamiya et al., 2003; Schuler et al., 2018; Wu et al., 2019). The deletion of OsSHRs will lead to the decrease of stomatal density in rice ( $\mathrm{Wu}$ et al., 2019), while the overexpression of ZmSHRs in rice produces additional stomatal files far away from the vein to increase stomatal density (Schuler et al., 2018). In the rsd1-1 mutant, we observed a decrease in the density of stomatal files in the leaf at the seedling stage (Figure 1L). However, the expression of OsSHR and OsSCR was not significantly changed in the rsd1 mutants compared with ZH11 (Figure 5), suggesting that the decrease of stomatal files in the mutants is independent of OsSHR/OsSCR.

Rice is one of the most important food crops in the world. Although global climatic variability is a serious threat to food security, genetic engineering of stomatal development will enable us to create stress-tolerant crops (Serna and Fenoll, 2002; Korres et al., 2017). By controlling stomatal development and reducing stomatal density, rice can control water loss and make it easier to survive under drought conditions (Buckley et al., 2019). The lack of RSD1 led to a reduction of stomatal density and the leaf water loss rate in rice. The effect of stomatal density on plants has been applied to create drought-resistant crops (Buckley et al., 2019). Recent research has shown that excessive expression of $E P F$ genes in wheat and rice can significantly improve water use efficiency without affecting plant yield when stomatal density is reduced (Caine et al., 2019; Dunn et al., 2019). Therefore,

\section{REFERENCES}

Abrash, E., Anleu Gil, M. X., Matos, J. L., and Bergmann, D. C. (2018). Conservation and divergence of YODA MAPKKK function in regulation of grass epidermal patterning. Development 145:dev165860. doi: 10.1242/dev. 165860

Abrash, E. B., and Bergmann, D. C. (2010). Regional specification of stomatal production by the putative ligand CHALLAH. Development 137, 447-455. doi: 10.1242/dev.040931

Berger, D., and Altmann, T. (2000). A subtilisin-like serine protease involved in the regulation of stomatal density and distribution in Arabidopsis thaliana. Genes Dev. 14, 1119-1131. doi: 10.1101/gad.14.9.1119

Bergmann, D. C., Lukowitz, W., and Somerville, C. R. (2004). Stomatal development and pattern controlled by a MAPKK kinase. Science 304, 14941497. doi: 10.1126/science.1096014

Bergmann, D. C., and Sack, F. D. (2007). Stomatal development. Annu. Rev. Plant Biol. 58, 163-181.

Bowman, J. L., Kohchi, T., Yamato, K. T., Jenkins, J., Shu, S., Ishizaki, K., et al. (2017). Insights into land plant evolution garnered from the Marchantia polymorpha Genome. Cell 171, 287.e15-304.e15. doi: 10.1016/j.cell.2017.09.030
RSD1 can be used as a candidate gene for breeding of droughtresistant rice.

\section{DATA AVAILABILITY STATEMENT}

The original contributions presented in the study are included in the article/Supplementary Material, further inquiries can be directed to the corresponding author.

\section{AUTHOR CONTRIBUTIONS}

QY and $\mathrm{SH}$ designed the experiments, and wrote the manuscript. QY, LC, WZ, TL, YA, ZW, YW, YX, and LY performed experiments, QY, LC, and SH revised the manuscript. All authors contributed to the article and approved the submitted version.

\section{ACKNOWLEDGMENTS}

We are grateful to Kunming Chen for kindly providing seeds of rel2 mutant. We appreciate the help of Dr. Yangwen Qian and Biogle Genetech Corporation in generating all CRISPR/Cas9 lines. This work was supported by the Ministry of Agriculture of the People's Republic of China (Grant No. 2016ZX08009003-002), the National Natural Science Foundation of China (NSFC) (Grant Nos. 31670185 and 31870251), the major project of Science and Technology of Gansu province (17ZD2NA016), and the Chang Jiang Scholars Program of China (2017).

\section{SUPPLEMENTARY MATERIAL}

The Supplementary Material for this article can be found online at: https://www.frontiersin.org/articles/10.3389/fpls.2020. 600021/full\#supplementary-material

Buckley, C. R., Caine, R. S., and Gray, J. E. (2019). Pores for thought: can genetic manipulation of stomatal density protect future rice yields? Front. Plant Sci. 10:1783. doi: 10.3389/fpls.2019.01783

Caine, R. S., Yin, X., Sloan, J., Harrison, E. L., Mohammed, U., Fulton, T., et al. (2019). Rice with reduced stomatal density conserves water and has improved drought tolerance under future climate conditions. New Phytol. 221, 371-384. doi: $10.1111 / \mathrm{nph} .15344$

Cartwright, H. N., Humphries, J. A., and Smith, L. G. (2009). A receptor-like protein that promotes polarization of an asymmetric cell division in maize. Science 323, 649-651. doi: 10.1126/science.1161686

Chen, L., Wu, Z., and Hou, S. (2020). SPEECHLESS speaks loudly in stomatal development. Front. Plant Sci. 11:114. doi: 10.3389/fpls.2020.00114

Dow, G. J., Berry, J. A., and Bergmann, D. C. (2014). The physiological importance of developmental mechanisms that enforce proper stomatal spacing in Arabidopsis thaliana. New Phytol. 201, 1205-1217. doi: 10.1111/nph.12586

Dunn, J., Hunt, L., Afsharinafar, M., Meselmani, M. A., Mitchell, A., Howells, R., et al. (2019). Reduced stomatal density in bread wheat leads to increased water-use efficiency. J. Exp. Bot. 70, 4737-4748. doi: 10.1093/jxb/erz248

Elert, E. (2014). Rice by the numbers: a good grain. Nature 514, S50-S51. doi: $10.1038 / 514$ S50a 
Facette, M. R., Park, Y., Sutimantanapi, D., Luo, A., Cartwright, H. N., Yang, B., et al. (2015). The SCAR/WAVE complex polarizes PAN receptors and promotes division asymmetry in maize. Nat. Plants 1:14024. doi: 10.1038/nplants.2014.24

Facette, M. R., and Smith, L. G. (2012). Division polarity in developing stomata. Curr. Opin. Plant Biol. 15, 585-592. doi: 10.1016/j.pbi.2012.09.013

Franks, P. J., and Farquhar, G. D. (1999). A relationship between humidity response, growth form and photosynthetic operating point in C3 plants. Plant Cell Environ. 22, 1337-1349. doi: 10.1046/j.1365-3040.1999 00494. x

Godfray, H. C. J., Beddington, J. R., Crute, I. R., Haddad, L., Lawrence, D., Muir, J. F., et al. (2010). Food security: the challenge of feeding 9 billion people. Science 327, 812-818. doi: 10.1126/science.1185383

Gonzalez-Mendoza, V., Zurita-Silva, A., Sanchez-Calderon, L., Sanchez-Sandoval, M. E., Oropeza-Aburto, A., Gutierrez-Alanis, D., et al. (2013). APSR1, a novel gene required for meristem maintenance, is negatively regulated by low phosphate availability. Plant Sci. 205-206, 2-12. doi: 10.1016/j.plantsci.2012. 12.015

Gordon, S. P., Contreras-Moreira, B., Levy, J. J., Djamei, A., Czedik-Eysenberg, A., Tartaglio, V. S., et al. (2020). Gradual polyploid genome evolution revealed by pan-genomic analysis of Brachypodium hybridum and its diploid progenitors. Nat. Commun. 11:3670. doi: 10.1038/s41467-020-17302-5

Hara, K., Kajita, R., Torii, K. U., Bergmann, D. C., and Kakimoto, T. (2007). The secretory peptide gene EPF1 enforces the stomatal one-cell-spacing rule. Genes Dev. 21, 1720-1725. doi: 10.1101/gad.1550707

Hara, K., Yokoo, T., Kajita, R., Onishi, T., Yahata, S., Peterson, K. M., et al. (2009). Epidermal cell density is autoregulated via a secretory peptide, EPIDERMAL PATTERNING FACTOR 2 in Arabidopsis leaves. Plant Cell Physiol. 50, 10191031. doi: $10.1093 /$ pcp/pcp068

Hetherington, A. M., and Woodward, F. I. (2003). The role of stomata in sensing and driving environmental change. Nature 424, 901-908. doi: 10.1038/ nature 01843

Hughes, J., Hepworth, C., Dutton, C., Dunn, J. A., Hunt, L., Stephens, J., et al. (2017). Reducing stomatal density in barley improves drought tolerance without impacting on yield. Plant Physiol. 174, 776-787. doi: 10.1104/pp.16. 01844

Hughes, T. E., Sedelnikova, O. V., Wu, H., Becraft, P. W., and Langdale, J. A. (2019). Redundant SCARECROW genes pattern distinct cell layers in roots and leaves of maize. Development 146:dev.177543. doi: 10.1242/dev.177543

Hunt, L., and Gray, J. E. (2009). The signaling peptide EPF2 controls asymmetric cell divisions during stomatal development. Curr. Biol. 19, 864-869. doi: 10. 1016/j.cub.2009.03.069

Kamiya, N., Itoh, J. I., Morikami, A., Nagato, Y., and Matsuoka, M. (2003). The SCARECROW gene's role in asymmetric cell divisions in rice plants. Plant J. 36, 45-54. doi: 10.1046/j.1365-313X.2003.01856.x

Kanaoka, M. M., Pillitteri, L. J., Fujii, H., Yoshida, Y., Bogenschutz, N. L., Takabayashi, J., et al. (2008). SCREAM/ICE1 and SCREAM2 specify three cellstate transitional steps leading to Arabidopsis stomatal differentiation. Plant Cell 20, 1775-1785. doi: 10.1105/tpc.108.060848

Korres, N., Norsworthy, J., Burgos, N., and Oosterhuis, D. (2017). Temperature and drought impacts on rice production: an agronomic perspective regarding short-and long-term adaptation measures. Water Resour. Rural Dev. 9, 12-27. doi: 10.1016/j.wrr.2016.10.001

Lai, L. B., Nadeau, J. A., Lucas, J., Lee, E. K., Nakagawa, T., Zhao, L., et al. (2005). The Arabidopsis R2R3 MYB proteins FOUR LIPS and MYB88 restrict divisions late in the stomatal cell lineage. Plant Cell 17, 2754-2767. doi: 10.1105/tpc.105. 034116

Lamesch, P., Berardini, T. Z., Li, D., Swarbreck, D., Wilks, C., Sasidharan, R., et al. (2012). The Arabidopsis information resource (TAIR): improved gene annotation and new tools. Nucleic Acids Res. 40, D1202-D1210. doi: 10.1093/ nar/gkr1090

Lampard, G. R., MacAlister, C. A., and Bergmann, D. C. (2008). Arabidopsis stomatal initiation is controlled by MAPK-mediated regulation of the bHLH SPEECHLESS. Science 322, 1113-1116. doi: 10.1126/science.1162263

Lang, D., Ullrich, K. K., Murat, F., Fuchs, J., Jenkins, J., Haas, F. B., et al. (2018). The Physcomitrella patens chromosome-scale assembly reveals moss genome structure and evolution. Plant J. 93, 515-533. doi: 10.1111/tpj.13801

Larkin, M. A., Blackshields, G., Brown, N. P., Chenna, R., McGettigan, P. A., McWilliam, H., et al. (2007). Clustal W and clustal X version 2.0. Bioinformatics 23, 2947-2948. doi: 10.1093/bioinformatics/btm404
Lau, O. S., and Bergmann, D. C. (2012). Stomatal development: a plant's perspective on cell polarity, cell fate transitions and intercellular communication. Development 139, 3683-3692. doi: 10.1242/dev.080523

Lee, E., Lucas, J. R., and Sack, F. D. (2014). Deep functional redundancy between FAMA and FOUR LIPS in stomatal development. Plant J. 78, 555-565. doi: 10.1111/tpj.12489

Lee, J. S., Hnilova, M., Maes, M., Lin, Y.-C. L., Putarjunan, A., Han, S.-K., et al. (2015). Competitive binding of antagonistic peptides fine-tunes stomatal patterning. Nature 522, 439-443. doi: 10.1038/nature14561

Lee, J. S., Kuroha, T., Hnilova, M., Khatayevich, D., Kanaoka, M. M., McAbee, J. M., et al. (2012). Direct interaction of ligand-receptor pairs specifying stomatal patterning. Genes Dev. 26, 126-136. doi: 10.1101/gad.179895.111

Letunic, I., and Bork, P. (2017). 20 years of the SMART protein domain annotation resource. Nucleic Acids Res. 46, D493-D496. doi: 10.1093/nar/gkx922

Letunic, I., and Bork, P. (2019). Interactive tree of life (iTOL) v4: recent updates and new developments. Nucleic Acids Res. 47, W256-W259. doi: 10.1093/nar/ gkz239

Liu, T., Ohashi-Ito, K., and Bergmann, D. C. (2009). Orthologs of Arabidopsis thaliana stomatal bHLH genes and regulation of stomatal development in grasses. Development 136, 2265-2276. doi: 10.1242/dev.032938

Liu, Y., Qin, L., Han, L., Xiang, Y., and Zhao, D. (2015). Overexpression of maize SDD1 (ZmSDD1) improves drought resistance in Zea mays L. by reducing stomatal density. Plant Cell Tiss. Organ Cult. 122, 147-159. doi: 10.1007/s11240015-0757-8

Lu, J., He, J., Zhou, X., Zhong, J., Li, J., and Liang, Y. K. (2019). Homologous genes of epidermal patterning factor regulate stomatal development in rice. J. Plant Physiol. 23, 18-27. doi: 10.1016/j.jplph.2019.01.010

Luo, L., Zhou, W. Q., Liu, P., Li, C. X., and Hou, S. W. (2012). The development of stomata and other epidermal cells on the rice leaves. Biol. Plant. 56, 521-527. doi: 10.1007/s10535-012-0045-y

MacAlister, C. A., Ohashi-Ito, K., and Bergmann, D. C. (2007). Transcription factor control of asymmetric cell divisions that establish the stomatal lineage. Nature 445, 537-540. doi: 10.1038/nature05491

McKown, K. H., and Bergmann, D. C. (2020). Stomatal development in the grasses: lessons from models and crops (and crop models). New Phytol. 227, 1636-1648. doi: $10.1111 /$ nph. 16450

Morales-Navarro, S., Perez-Diaz, R., Ortega, A., de Marcos, A., Mena, M., Fenoll, C., et al. (2018). Overexpression of a SDD1-Like gene from wild tomato decreases stomatal density and enhances dehydration avoidance in Arabidopsis and cultivated Tomato. Front. Plant Sci. 9:940. doi: 10.3389/fpls.2018.00940

Nishimura, A., Aichi, I., and Matsuoka, M. (2006). A protocol for Agrobacteriummediated transformation in rice. Nat. Protoc. 1, 2796-2802. doi: 10.1038/nprot. 2006.469

Niwa, T., Kondo, T., Nishizawa, M., Kajita, R., Kakimoto, T., and Ishiguro, S. (2013). EPIDERMAL PATTERNING FACTOR LIKE5 peptide represses stomatal development by inhibiting meristemoid maintenance in Arabidopsis thaliana. Biosci. Biotechnol. Biochem. 77, 1287-1295. doi: 10.1271/bbb.1 30145

Nunes, T. D. G., Zhang, D., and Raissig, M. T. (2020). Form, development and function of grass stomata. Plant J. 101, 780-799. doi: 10.1111/tpj.14552

Ohashi-Ito, K., and Bergmann, D. C. (2006). Arabidopsis FAMA controls the final proliferation/differentiation switch during stomatal development. Plant Cell 18, 2493-2505. doi: 10.1105/tpc.106.046136

Olsen, J. L., Rouze, P., Verhelst, B., Lin, Y. C., Bayer, T., Collen, J., et al. (2016). The genome of the seagrass Zostera marina reveals angiosperm adaptation to the sea. Nature 530, 331-335. doi: 10.1038/nature16548

Ouyang, S., Zhu, W., Hamilton, J., Lin, H., Campbell, M., Childs, K., et al. (2007). The TIGR rice genome annotation resource: improvements and new features. Nucleic Acids Res. 35, D883-D887. doi: 10.1093/nar/gk1976

Pillitteri, L. J., Sloan, D. B., Bogenschutz, N. L., and Torii, K. U. (2007). Termination of asymmetric cell division and differentiation of stomata. Nature 445, 501-505. doi: 10.1038/nature05467

Putarjunan, A., Ruble, J., Srivastava, A., Zhao, C., Rychel, A. L., Hofstetter, A. K., et al. (2019). Bipartite anchoring of SCREAM enforces stomatal initiation by coupling MAP kinases to SPEECHLESS. Nat. Plants 5, 742-754. doi: 10.1038/ s41477-019-0440-x

Qi, X., and Torii, K. U. (2018). Hormonal and environmental signals guiding stomatal development. BMC Biol. 16:21. doi: 10.1186/s12915-018-0488-5 
Qu, X., Yan, M., Zou, J., Jiang, M., Yang, K., and Le, J. (2018). A2-type cyclin is required for the asymmetric entry division in rice stomatal development. J. Exp. Bot. 69, 3587-3599. doi: 10.1093/jxb/ery158

Raissig, M. T., Abrash, E., Bettadapur, A., Vogel, J. P., and Bergmann, D. C. (2016). Grasses use an alternatively wired bHLH transcription factor network to establish stomatal identity. Proc. Natl. Acad. Sci. U.S.A. 113, 8326-8331. doi: $10.1073 /$ pnas.1606728113

Raissig, M. T., Matos, J. L., Gil, M. X. A., Kornfeld, A., Bettadapur, A., Abrash, E., et al. (2017). Mobile MUTE specifies subsidiary cells to build physiologically improved grass stomata. Science 355, 1215-1218. doi: 10.1126/science.aal3254

Saitou, N., and Nei, M. (1987). The neighbor-joining method: a new method for reconstructing phylogenetic trees. Mol. Biol. Evol. 4, 406-425. doi: 10.1093/ oxfordjournals.molbev.a040454

Schnable, P. S., Ware, D., Fulton, R. S., Stein, J. C., Wei, F., Pasternak, S., et al. (2009). The B73 maize genome: complexity, diversity, and dynamics. Science 326:1112. doi: 10.1126/science.1178534

Schuler, M. L., Sedelnikova, O. V., Walker, B. J., Westhoff, P., and Langdale, J. A. (2018). SHORTROOT-mediated increase in stomatal density has no impact on photosynthetic efficiency. Plant Physiol. 176, 757-772. doi: 10.1104/pp.17. 01005

Serna, L., and Fenoll, C. (2002). Reinforcing the idea of signaling in the stomatal pathway. Trends Genet. 18, 597-600. doi: 10.1016/s0168-9525(02)02790-7

Shpak, E. D., McAbee, J. M., Pillitteri, L. J., and Torii, K. U. (2005). Stomatal patterning and differentiation by synergistic interactions of receptor kinases. Science 309, 290-293. doi: 10.1126/science.1109710

Slewinski, T. L., Anderson, A. A., Price, S., Withee, J. R., Gallagher, K., and Turgeon, R. (2014). Short-root1 plays a role in the development of vascular tissue and kranz anatomy in maize leaves. Mol. Plant 7, 1388-1392. doi: 10.1093/mp/ ssu036

Stebbins, G., and Shah, S. (1960). Developmental studies of cell differentiation in the epidermis of monocotyledons: II. Cytological features of stomatal development in the Gramineae. Dev. Biol. 2, 477-500. doi: 10.1016/00121606(60)90050-6

Sugano, S. S., Shimada, T., Imai, Y., Okawa, K., Tamai, A., Mori, M., et al. (2010). Stomagen positively regulates stomatal density in Arabidopsis. Nature 463, 241-244. doi: 10.1038/nature08682

Tomato Genome, C. (2012). The tomato genome sequence provides insights into fleshy fruit evolution. Nature 485, 635-641. doi: 10.1038/nature11119

Vogel, J. P., Garvin, D. F., Mockler, T. C., Schmutz, J., Rokhsar, D., Bevan, M. W., et al. (2010). Genome sequencing and analysis of the model grass Brachypodium distachyon. Nature 463, 763-768. doi: 10.1038/nature08747
Von Groll, U., Berger, D., and Altmann, T. (2002). The subtilisin-like serine protease SDD1 mediates cell-to-cell signaling during Arabidopsis stomatal development. Plant Cell 14, 1527-1539. doi: 10.1105/tpc.001016

Wang, H., Guo, S., Qiao, X., Guo, J., Li, Z., Zhou, Y., et al. (2019). BZU2/ZmMUTE controls symmetrical division of guard mother cell and specifies neighbor cell fate in maize. PLoS Genet. 15:e1008377. doi: 10.1371/journal.pgen.1008377

Wang, H., Ngwenyama, N., Liu, Y., Walker, J. C., and Zhang, S. (2007). Stomatal development and patterning are regulated by environmentally responsive mitogen-activated protein kinases in Arabidopsis. Plant Cell 19, 63-73. doi: 10.1105/tpc.106.048298

Wu, Z., Chen, L., Yu, Q., Zhou, W., Gou, X., Li, J., et al. (2019). Multiple transcriptional factors control stomata development in rice. New Phytol. 223, 220-232. doi: 10.1111/nph.15766

Xie, X., Ma, X., Zhu, Q., Zeng, D., Li, G., and Liu, Y.-G. (2017). CRISPR-GE: a convenient software toolkit for CRISPR-based genome editing. Mol. Plant 10, 1246-1249. doi: 10.1016/j.molp.2017.06.004

Xu, N., Wang, R., Zhao, L., Zhang, C., Li, Z., Lei, Z., et al. (2016). The Arabidopsis NRG2 protein mediates nitrate signaling and interacts with and regulates key nitrate regulators. Plant Cell 28, 485-504. doi: 10.1105/tpc.15.00567

Yang, S. Q., Li, W. Q., Miao, H., Gan, P. F., Qiao, L., Chang, Y. L., et al. (2016). REL2, a gene encoding an unknown function protein which contains DUF630 and DUF632 domains controls leaf rolling in rice. Rice 9:37. doi: 10.1186/s12284016-0105-6

Young, N. D., Debelle, F., Oldroyd, G. E., Geurts, R., Cannon, S. B., Udvardi, M. K., et al. (2011). The Medicago genome provides insight into the evolution of rhizobial symbioses. Nature 480, 520-524. doi: 10.1038/nature10625

Zwieniecki, M. A., and Boyce, C. K. (2014). Evolution of a unique anatomical precision in angiosperm leaf venation lifts constraints on vascular plant ecology. Proc. Biol. Sci. 281:20132829. doi: 10.1098/rspb.2013.2829

Conflict of Interest: The authors declare that the research was conducted in the absence of any commercial or financial relationships that could be construed as a potential conflict of interest.

Copyright (c) $2020 \mathrm{Yu}$, Chen, Zhou, An, Luo, Wu, Wang, Xi, Yan and Hou. This is an open-access article distributed under the terms of the Creative Commons Attribution License (CC BY). The use, distribution or reproduction in other forums is permitted, provided the original author(s) and the copyright owner(s) are credited and that the original publication in this journal is cited, in accordance with accepted academic practice. No use, distribution or reproduction is permitted which does not comply with these terms. 